\title{
Purely Organic Room-Temperature Phosphorescence Endowing Fast Intersystem Crossing from Through-Space Spin-Orbit Coupling
}

Jie Yu, ${ }^{\bigcirc}$ Huili Ma, ${ }^{\bigcirc}$ Wenbin Huang, Zhiwei Liang, Kang Zhou, Anqi Lv, Xin-Gui Li, ${ }^{*}$ and Zikai He*

Cite This: JACS Au 2021, 1, 1694-1699

Read Online

ABSTRACT: Purely organic room-temperature phosphorescence endowing very fast intersystem crossing from through-space systems has not been well investigated. Here we report three space-confined bridged phosphors, where phenothiazine is linked with dibenzothiophene, dibenzofuran, and carbazole by a 9,9-dimethylxanthene bridge. Nearly pure phosphorescence is observed in the crystals at room temperature. Interestingly, phosphorescence comes solely from the phenothiazine segment. Experimental results indicate that bridged counterparts of dibenzothiophene, dibenzofuran, and carbazole contribute as close-lying triplet states with locally excited (LE) character. The throughspace spin-orbit coupling principle is proposed in these bridged systems, as their ${ }^{1} \mathrm{LE}$ and ${ }^{3} \mathrm{LE}$ states have intrinsic spatial overlap, degenerate energy levels, and tilting face-to-face alignment. The resulting effective through-space spin-orbit coupling leads to efficient intersystem crossing a with rate constant as high as $10^{9} \mathrm{~s}^{-1}$ and an overwhelming triplet decay channel of the singlet excited state.

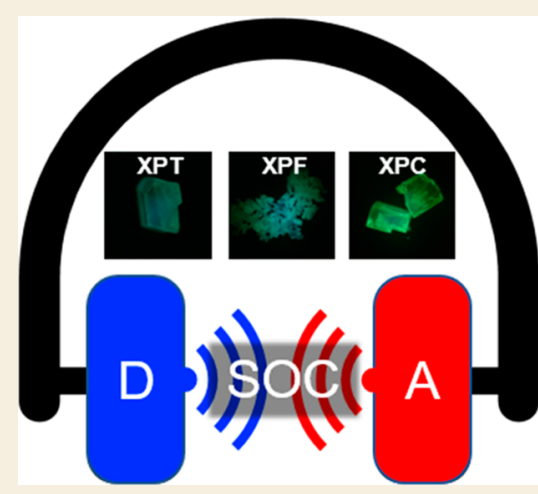

KEYWORDS: room-temperature phosphorescence, through-space interaction, spin-orbit coupling, triplet exciton, solid-state photophysics

Tntersystem crossing (ISC), originally considered forbidden in purely organic materials, can become very fast when the effective spin-orbit coupling (SOC) principle works. ${ }^{1}$ It is well-known that the El-Sayed rule, ${ }^{2}$ heavy-atom effect, ${ }^{3,4}$ and energy gap law ${ }^{5}$ can induce such effective SOC for spin flipping between singlet and triplet excitons. Years ago, as an important property of triplet excitons, room-temperature phosphorescence (RTP) was obtained by stabilizing the sensitive triplet excitons through several classic protocols, ${ }^{6}$ including the pioneering work of micelle formation, ${ }^{7,8}$ host-guest matrix, ${ }^{9}$ polymer aggregation, ${ }^{10-12}$ crystallization, ${ }^{13-17}$ halogen bonding, ${ }^{18}$ and self-assembly. ${ }^{19}$ Recently, RTP has received significant attention $^{20,21}$ for its exceptional applications in newly emerged technologies such as chemical sensors, ${ }^{22,23}$ anticounterfeiting, ${ }^{24}$ optical recording, ${ }^{25}$ lighting and display, ${ }^{26}$ and bioimaging. ${ }^{27-29}$ However, it is still an enormous challenge to realize pure RTP in organic molecules without heavy atoms. ${ }^{30}$ Importantly, ISC determines the upper limit of RTP efficiency. As the SOC principle is dominant for achieving a fast ISC process, boosted triplet population, and efficient RTP emission, exploring an effective SOC principle becomes highly desirable. ${ }^{31}$

Recently, through-space charge transfer has been an alternative to realize efficient thermally activated delayed fluorescence. $^{32-38}$ A reverse ISC channel is greatly enhanced through a tunable small energy gap between singlet and triplet states $\left(\Delta E_{\mathrm{ST}}\right)$ and sufficient electronic coupling between donor and acceptor motifs. In addition, rigid and closely packed coplanar configurations suppress nonradiative decay, resulting in high photoluminescent quantum yields. ${ }^{39}$ However, as the counterpart, the effect on the forward ISC channel has not been well investigated. ${ }^{40}$

Here, we designed and synthesized three space-confined bridged phosphors (Figure 1). The unconjugated 9,9dimethylxanthene bridge links three weak electronic D-A counterparts (D, phenothiazine; ${ }^{41} \mathrm{~A}$, dibenzothiophene, dibenzofuran, and carbazole $\mathrm{e}^{42-44}$ ). Experimental and theoretical investigations determined that the spatial $\pi-\pi$ interaction mediates weak through-space charge-transfer character and nearly pure phosphorescence is achieved in crystals at room temperature by a overwhelming competition against fluorescence. The rate constant of ISC is found to be as high as $10^{9}$ $\mathrm{s}^{-1}$. This improvement in ISC can be ascribed to characters of the singlet and triplet excitons involved that have degenerate energy levels, locally excited (LE) characters, and intrinsic spatial overlap. Thus, a through-space SOC principle is put forth in these bridged weak D-A molecular structures. Furthermore, the molecular structure offers a high tunability of the backbone, distance, and orientation of $\mathrm{D}$ and $\mathrm{A}$ segments to study this interesting SOC principle in the future.

Received: June 27, 2021

Published: September 14, 2021 

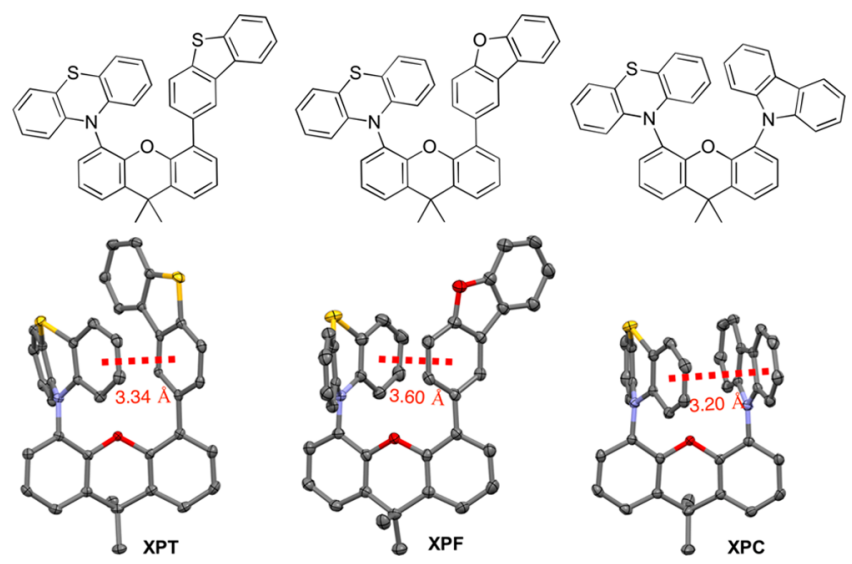

Figure 1. Molecular and single-crystal structures of XPT, XPF, and $\mathrm{XPC}$, together with the shortest $\pi-\pi$ distances. Carbon, oxygen, nitrogen, and sulfur atoms are shown as gray, red, blue, and yellow ellipsoids, respectively, at the $50 \%$ probability level.

Three aromatic through-space bridged compounds, 10-(5(dibenzo[b,d] thiophen-2-yl)-9,9-dimethyl-9H-xanthen-4-yl)$10 H$-phenothiazine (XPT), 10-(5-(dibenzo[b,d]furan-2-yl)9,9-dimethyl-9H-xanthen-4-yl)-10H-phenothiazine (XPF), and 10-(5-(9H-carbazol-9-yl)-9,9-dimethyl-9H-xanthen-4-yl)$10 H$-phenothiazine (XPC) have been synthesized through Suzuki-Miyaura and Ullman coupling reactions accordingly (Scheme S1). All of the molecules have been characterized by ${ }^{1} \mathrm{H}$ and ${ }^{13} \mathrm{C}$ NMR spectroscopy (Charts S1-S6), highresolution mass spectra (Charts S7-S9), differential scanning calorimetry, thermogravimetric analysis (Charts S10-S12), and X-ray single-crystal structure analyses (Tables S1-S3). CCDC 2091147, 2091148, and 2091149 contain supplementary crystallographic data for this paper. These data can be obtained free of charge via www.ccdc.cam.ac.uk/data_request/ cif, or by emailing data_request@ccdc.cam.ac.uk, or by contacting The Cambridge Crystallographic Data Centre, 12 Union Road, Cambridge CB2 1EZ, UK; fax: + 441223 336033. The carbazole starting material was synthesized in our laboratory, and the products were purified by column chromatography and three recrystallizations, removing the possible effects from impurities discovered recently. ${ }^{44-46}$ The crystal structures shown in Figure 1 confirm the nearly cofacial arrangement of the donor and acceptor phenyl groups, with the shortest $\pi-\pi$ distance being around $3.20-3.60 \AA$. It is worth noting that the segment orientation has an overall tilting face-to-face alignment, providing the possibility for good electronic coupling, particularly at excited states. In comparison, there is no intermolecular $\pi-\pi$ stacking in the crystals (Figure S1).

The fundamental photophysical properties of XPT are first studied as representative. The absorption spectrum clearly reflects the sum of xanthene, phenothiazine, and dibenzothiophene contributions at short wavelength (Figure 2a). Surprisingly, XPT shows UV-vis spectra devoid of the tail absorption of phenothiazine at long wavelengths, indicating negligible conjugation across the donor-acceptor units and thus complete electronic decoupling in the ground state. The space-confined conformation makes the vibration of phenothiazine difficult at the ground state, which accounts for its tail absorption and the vanishing absorption in XPT. Figure $2 b, c$ shows the steady-state (prompt) and time-resolved (delayed 1 $\mathrm{ms}$ ) photoluminescence (PL) spectra of the crystalline
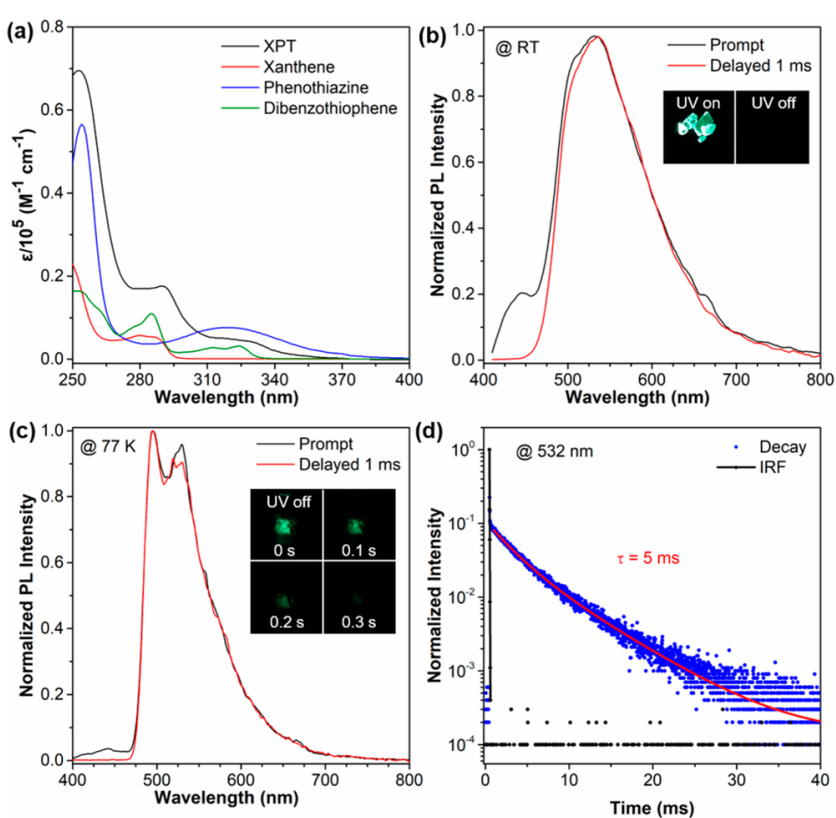

Figure 2. Absorption and photoluminescence spectra of XPT. (a) UV-visible absorption spectra of XPT, xanthene, phenothiazine, and dibenzothiophene. (b) PL spectra of an XPT crystal at room temperature. Inset: pictures of XPT under $365 \mathrm{~nm}$ UV irradiation and with UV irradiation off at room temperature. (c) PL spectra of an XPT crystal measured at $77 \mathrm{~K}$. Inset: pictures of UV irradiation off at $0 \mathrm{~s}, 0.1 \mathrm{~s}, 0.2 \mathrm{~s}$, and $0.3 \mathrm{~s}$ at $77 \mathrm{~K}$. (d) Decay curve and lifetime of an XPT crystal at room temperature detected at $532 \mathrm{~nm}$. The excitation wavelength was $380 \mathrm{~nm}\left(\tau_{1}=2.6 \mathrm{~ms}, \tau_{2}=6.2 \mathrm{~ms}\right.$; the amounts of $\tau_{1}$ and $\tau_{2}$ are $34 \%$ and $66 \%$, respectively).

powders at room temperature and $77 \mathrm{~K}$, respectively. The perfect overlapping between prompt and delayed spectra indicates that the phosphorescence emission dominates the overall PL intensity. Inset pictures exhibit the same emission color among different states with different delay times (Figure S2a), further proving the pure phosphorescence characteristics. However, the solutions and amorphous powders are nonemissive and only emit fluorescence, respectively, at room temperature (Figure S2b,c). In addition, different afterglow features are found for an ultrashort lifetime at room temperature and a long lifetime at $77 \mathrm{~K}$. To verify this, the time-resolved decay curves at $532 \mathrm{~nm}$ were recorded (Figure $2 \mathrm{~d}$ ). The average lifetimes are found to be $5 \mathrm{~ms}$ for crystals at room temperature and $77 \mathrm{~ms}$ for crystals at $77 \mathrm{~K}$ (Figure S3). The temperature-dependent PL spectra and decay curves are shown in Figures S4-S6. The intensity of dominating phosphorescence from 450 to $700 \mathrm{~nm}$ increases with a decrease in temperature, while the intensity and lifetime of weak fluorescence from 400 to $450 \mathrm{~nm}$ is not influenced. At low temperature, the molecular vibration and diffusion can be effectively suppressed and nonradiative decays are reduced, which is beneficial for stabilizing excited states and light emission. The phosphorescence shows an obvious temperature-dependent characteristic. However, the unaffected fluorescence is because of the overwhelming rate constant of the ISC process over that of the nonradiative decay of $S_{1}$ regardless of temperature. Due to the high rigidity of the molecular structure in the crystalline environment at low temperature, the PL spectral profile remains unchanged despite the intensity. 
Detailed PL spectra and decay curves reveal that the phosphorescence of XPT solely stems from the phenothiazine segment (Figure 3). Phenothiazine emits pure fluorescence in the crystalline and solution states at room temperature; its phosphorescence is only detected at $77 \mathrm{~K}$ (Figure 3a,b). The fact indicates the possible heavy-atom effect of an $S$ atom in comparison with pure hydrocarbon compounds. However, the accurate effect needs further study. In comparison, it is noted that the XPT crystal and solution both emit phosphorescence profiles quite similar in shape with that of phenothiazine solution rather than its crystal, which indicates that XPT inherits the molecular properties of phenothiazine rather than its aggregate. The difference in emission wavelength can be attributed to the different surrounding polarities in the crystal and solution. It is seen that the XPT crystal endows confined and isolated surroundings to the phenothiazine segment, which contributes to the phosphorescence emission. Time-resolved decay curves confirm that the phosphorescence lifetimes of $\mathrm{XPT}$ and phenothiazine lie in the same region. Moreover, temperature-dependent decay curves show that the XPT crystal enjoys a decrease in lifetime with an increase in temperature, which removes the possibility of thermally activated delayed fluorescence. A careful comparison of PL spectra and decay curves of dibenzothiophene, xanthene, and $\mathrm{XPT}$ excludes the possibility that phosphorescence of XPT comes from these segments (Figures S7-S12). They are dramatically different in emission profiles, wavelength, and lifetimes. Why does only the phenothiazine segment contribute to the pure phosphorescence emission of XPT?

To answer this question, we measured the photophysical properties of XPF and XPC for comparison (Table 1 and Figures S13-S24). They exhibit behaviors quite similar to that of XPT. It is found that both compounds present
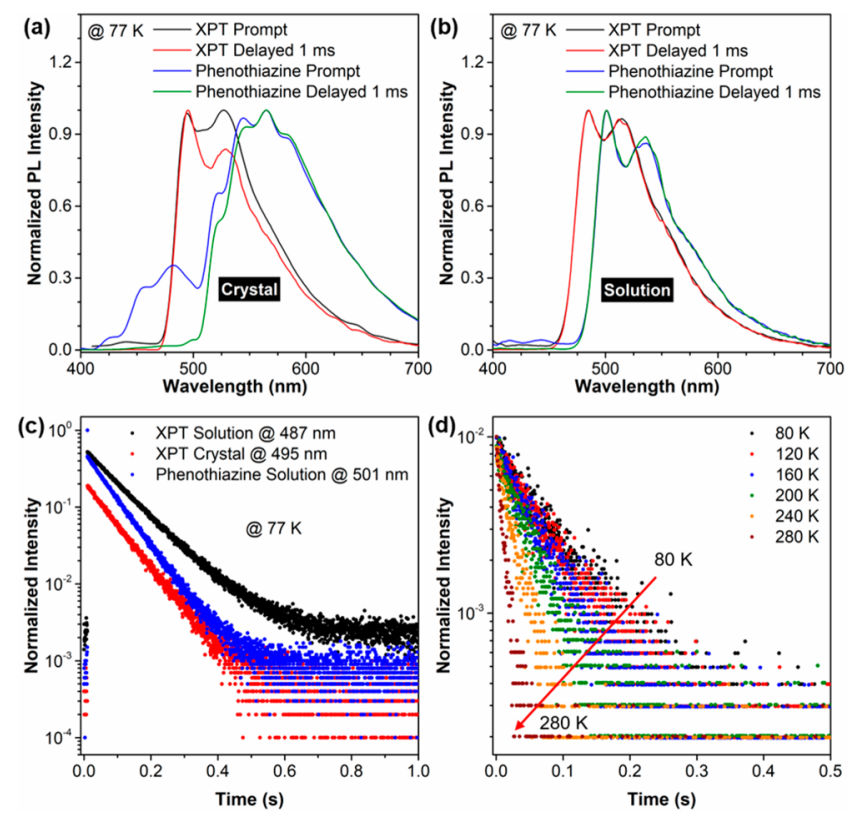

Figure 3. (a) PL spectra of an XPT crystal and a phenothiazine crystal at $77 \mathrm{~K}$. (b) PL spectra of XPT and phenothiazine in 2-Me-THF $\left(10^{-5} \mathrm{M}\right)$ at $77 \mathrm{~K}$. (c) Lifetime of an XPT crystal and phenothiazine in the crystal form and in 2-Me-THF solution $\left(10^{-5} \mathrm{M}\right)$. (d) Lifetime of an XPT crystal at $495 \mathrm{~nm}$ at different temperatures from 80 to 280 $\mathrm{K}$. The excitation wavelengths are $380 \mathrm{~nm}$ for an XPT crystal and 320 $\mathrm{nm}$ for an XPT solution and phenothiazine.
Table 1. Photophysical Properties of XPT, XPF, and XPC ${ }^{a}$

$\begin{array}{lcccccc} & \lambda_{\mathrm{PL}}(\mathrm{nm}) & \begin{array}{c}\Phi_{\mathrm{PL}} \\ (\%)\end{array} & \begin{array}{c}\text { P:F } \\ \text { ratio }\end{array} & \tau_{\mathrm{F}}(\mathrm{ns}) & \tau_{\mathrm{P}}(\mathrm{ms}) & k_{\mathrm{ISC}}\left(10^{8} \mathrm{~s}^{-1}\right) \\ \mathrm{XPT} & 433,514 & 4.25 & 31: 1 & 1.41 & 5.00 & 7.1 \\ \mathrm{XPF} & 444,510 & 2.02 & 6.4: 1 & 1.03 & 1.12 & 9.7 \\ \mathrm{XPC} & 447,513 & 3.53 & 125: 1 & 0.83 & 15.18 & 12.0\end{array}$

${ }^{a}$ Crystal samples were measured at room temperature. The P:F ratio denotes the phosphorescence:fluorescence ratio by carefully separating their contributions to the overall PL spectra.

phosphorescence profiles almost identical with that of XPT in their crystals at room temperature and in solution at $77 \mathrm{~K}$ (Figure 4a and Figures S25-S28), indicating that all of their phosphorescence stems from the phenothiazine segment and that the bridged segments of dibenzofuran, dibenzothiophene, and carbazole have little influence on the $\mathrm{T}_{1}$ states. The quantum yields are moderate for these three phosphors but are much lower than that of the reported fluorescent system. The possible reasons are the feasible vibration of nonplanar phenothiazine in the bridged molecular conformation and a similar promotion by through-space spin-orbit coupling in the nonradiative decay of $T_{1}$ to $S_{0}$. Nevertheless, this is a crucial problem at this stage, which requires further investigation to enhance the phosphorescence efficiency. In addition, we note that the ratios of their phosphorescence are diverse (Figure S29). Nevertheless, the phosphorescence still dominates the emission from $86 \%$ to $96 \%$ in these three phosphors (Figures S30-S32). Together with fluorescence and phosphorescence lifetimes, the decay rate constants of ISC $\left(k_{\text {ISC }}\right)$ are estimated to be as high as $(7-12) \times 10^{8} \mathrm{~s}^{-1}$, much larger than those of the reported heavy-atom-free RTP systems ${ }^{47}\left(0.12-2.2 \times 10^{8}\right.$ $\mathrm{s}^{-1}$ ) and the reverse ISC of covalent bonding D-A type thermally activated delayed fluorescence systems ${ }^{48}\left(1.2 \times 10^{7}\right.$ $\left.\mathrm{s}^{-1}\right)$.

To decipher the working mechanism, the energy levels of the $S_{1}$ and $T_{1}$ states of the segments and the bridged molecules have been carefully estimated from their low-temperature fluorescence and phosphorescence spectra (Figure 3a,b and Figures S7, S8, S25-S28, and S33-S38). As summarized in Figure $4 \mathrm{~d}$, the integrated molecules basically have energy levels inherited from the corresponding low-lying model segments, following the classic Kasha rule and indicating the weak electronic coupling between these segments. In a word, the integrated molecules of XPT, XPF, and XPC show quite similar PL spectra in rigid-glass solution and the crystal form at $77 \mathrm{~K}$, suggesting that all their $\mathrm{T}_{1}$ states are low-lying locally excited triplet $\left({ }^{3} \mathrm{LE}\right)$ states of the phenothiazine unit. It is worth noting that the energy levels of $S_{1}\left({ }^{1} \mathrm{LE}_{\mathrm{PTZ}}\right)$ of the phenothiazine molecule and $\mathrm{T}_{n}\left({ }^{3} \mathrm{LE}_{\mathrm{DBT}},{ }^{3} \mathrm{LE}_{\mathrm{DBF}},{ }^{3} \mathrm{LE}_{\mathrm{CZ}}\right)$ are quite close, giving a very small $\Delta E_{\mathrm{ST}}$ for the energy gap law to work in the ISC decay channel (Figure 4d).

As is well-known, a small $\Delta E_{\mathrm{ST}}$ can be easily achieved by separating the highest occupied molecular orbital (HOMO) and the lowest unoccupied molecular orbital (LUMO) in charge-transfer (CT) systems of covalent bonding of electron donor and acceptor units. The situation remains the same in our through-space bridged systems. The difference lies in the following. Covalently bonded D-A systems require a significant electronic push-pull effect to dramatically separate the HOMO and LUMO spatial distribution because $\pi$ segments are feasible for electronic coupling through bonds. However, our through-space bridged systems are insensitive to the 

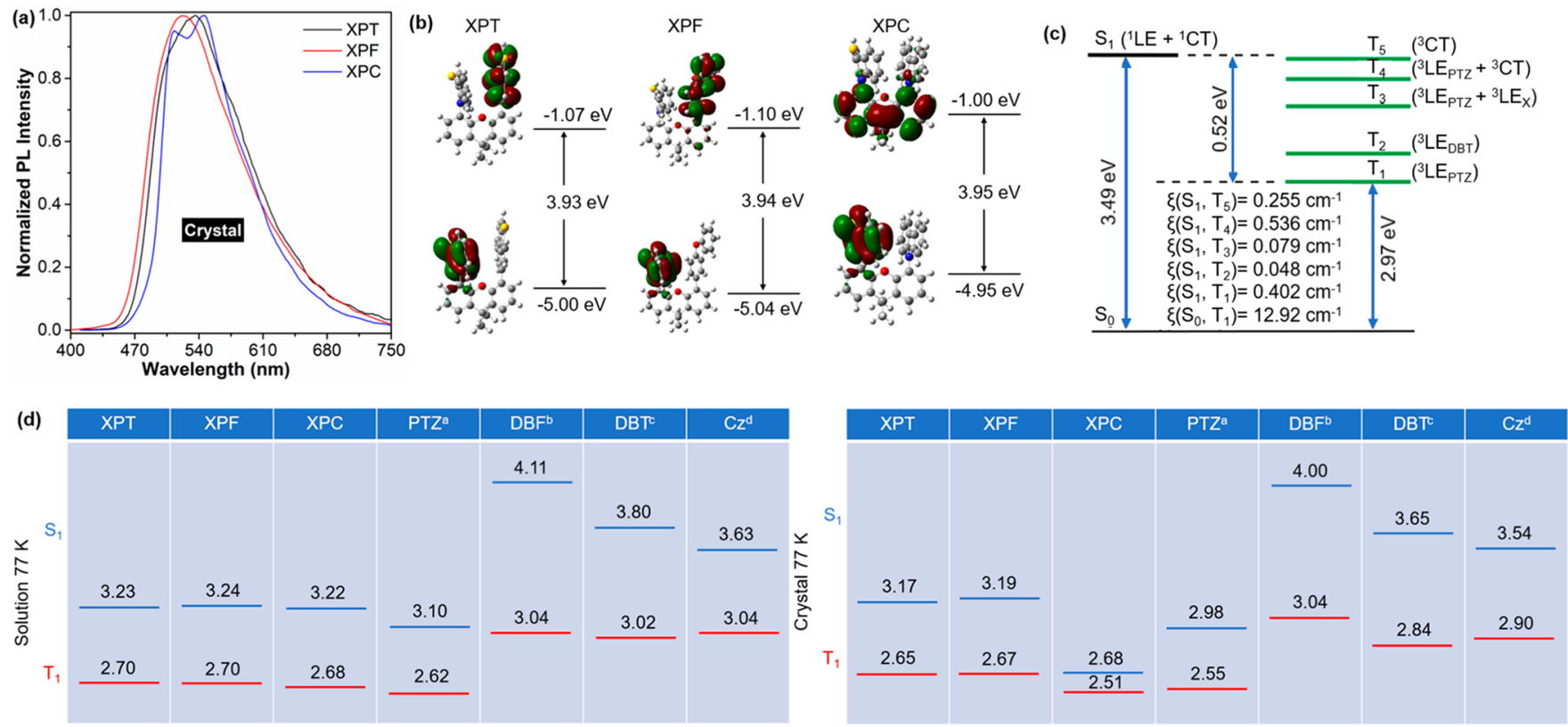

Figure 4. (a) Delayed phosphorescence spectra of XPF, XPT, and XPC crystals at room temperature. (b) HOMO and LUMO orbital distribution and calculated band gaps of XPT, XPF, and XPC based on TD-DFT with the B3LYP functional and 6-31G* basis set. (c) Calculated energy diagram and spin-orbit coupling (SOC) matrix element values of XPT. PTZ, DBF, DBT, Cz, and X stand for phenothiazine, dibenzofuran, dibenzothiophene, carbazole, and xanthene, respectively. (d) Energy levels of excited states of XPF, XPT, XPC, and their model fragments.

electronic features of the counterparts as they are intrinsically far away in space, particularly at the ground state. As a result, a small difference in electronic properties can endow an obvious HOMO and LUMO separation, giving a high tunability of the backbone of $\mathrm{D}$ and $\mathrm{A}$ units. The slight structure derivation can fulfill the requirement for the HOMO-LUMO separation such as in XPT, XPF, and XPC. The calculated distributions of HOMO and LUMO support our descriptions in Figure $4 \mathrm{~b}$, and the energy levels are also perfectly validated by cyclic voltammetry in Figures S39-S41. In a word, the small spatial overlap of frontier orbitals results in a negligible $\Delta E_{\mathrm{ST}}$ value in both through-bond and through-space $\mathrm{D}-\mathrm{A}$ systems.

However, the energy gap law cannot fully explain the sole phosphorescence from phenothiazine. Particularly, the spin-flip process of ISC between $S_{1}$ and $T_{1}$ states having the same configuration character is theoretically spin forbidden according to the El-Sayed rules because of the weak SOC matrix element. ${ }^{48}$ The situation often occurs in CT-type thermally activated delayed fluorescence systems. Recently, the inclusion of ${ }^{3} \mathrm{LE}$ has been reported to accelerate reverse ISC. The inherently forbidden reverse ISC transition becomes effective when the ${ }^{3} \mathrm{LE}$ bridged state lies in the vibronic range of energy levels between ${ }^{1} \mathrm{CT}$ and ${ }^{3} \mathrm{CT} .{ }^{49}$ As shown in Figure $4 \mathrm{c}$ and Figure S42, high-lying triplet excited states $\mathrm{T}_{n}$ and the related $S_{1}$ state often enjoy hybrid orbital configurations that contain both $\mathrm{CT}$ and LE characters and thus benefit from the spinvibronic coupling mechanism. It is worth noting that $S_{0}-T_{1}$ gives the greatest value of the SOC matrix element, which explains the quite low quantum yield and short lifetime of PTZ-containing phosphors.

Here, we propose another design principle to realize an ideal system that exhibits highly efficient ISC, that in a throughspace bridged weak D-A scaffold. Weak D and A $\pi$ segments (quite similar in electronic properties) are connected through a bridge at a suitable distance. Under weak electronic coupling, $\mathrm{S}_{1}$ and close-lying $\mathrm{T}_{n}$ states can contain LE character contributed both by $\mathrm{D}$ and A $\pi$ segments. Therefore, these ${ }^{1} \mathrm{LE}$ and ${ }^{3} \mathrm{LE}$ excitons could enjoy good spatial overlap in excited states. Versatile $\mathrm{D} / \mathrm{A}$ structure candidates allow degenerate energy levels of $S_{1}$ and $T_{n}$ for applications of the energy gap law. Finally, the tilting face-to-face alignment of the D/A segment makes "out of co-plane" vibration possible to allow mixing of ${ }^{1} \mathrm{LE}$ and ${ }^{3} \mathrm{LE}$ states. ${ }^{50}$ Overall, the spin-orbit coupling has considerable matrix element values to achieve effective SOC coupling during the ISC process in these heavyatom-free through-space bridged systems. Furthermore, other kinds of functional $\pi$ segmens can also be easily introduced into the scaffold. Such an expansion will provide new RTP materials with improved performance and give insights into the fundamental photophysical properties to study this interesting SOC principle thoroughly. Interestingly, enhanced SOC normally leads to boosted phosphorescent and nonradiative decay of $T_{1}$, which is expected to shorten the phosphorescent lifetime, which is not the case here. The possible reason lies in the intermolecular triplet-triplet energy transfer. ${ }^{43}$ The energy transfer successfully separates the locations of the initially populated $\mathrm{T}_{n}$ center $\left({ }^{3} \mathrm{LE}_{\mathrm{DBT}},{ }^{3} \mathrm{LE}_{\mathrm{DBF}},{ }^{3} \mathrm{LE}_{\mathrm{CZ}}\right)$ and the finally emissive $\mathrm{T}_{1}$ center $\left({ }^{3} \mathrm{LE}_{\mathrm{PTZ}}\right)$, allowing simultaneous achievement of a high $k_{\text {ISC }}$ to enhance the efficiency and maintain $k_{\mathrm{P}}$ and $k_{\mathrm{nr}}$ to a low level to prolong the lifetime. Therefore, regardless of the bridges, the phosphors maintain the lifetime originating from phenothiazine.

In conclusion, we have put forth a new design strategy for an RTP system with a through-space bridged weak D-A motif. Experimental evidence and theoretical simulations demonstrate that the SOC process occurs within D-A segments. Spatially and energetically close lying excited states with LE character result in the considerable SOC matrix element value, giving an overwhelming ISC decay of singlet excited state. Furthermore, the molecular structure allows a high tunability of $\mathrm{D}$ and $\mathrm{A}$ segments with various types, numbers, and 
combinations to expand the system and explore functional phosphors.

\section{ASSOCIATED CONTENT}

Supporting Information

The Supporting Information is available free of charge at https://pubs.acs.org/doi/10.1021/jacsau.1c00290.

Materials, instruments, synthetic and experimental procedures, and characterization data (PDF)

\section{AUTHOR INFORMATION}

\section{Corresponding Authors}

Xin-Gui Li - State Key Laboratory of Pollution Control and Resource Reuse, and Shanghai Institute of Pollution Control and Ecological Security, College of Environmental Science and Engineering, Tongji University, Shanghai 200092, People's Republic of China; Email: lixingui@tongji.edu.cn

Zikai He - School of Science, Harbin Institute of Technology, Shenzhen, Guangdong 518055, People's Republic of China; School of Chemical Engineering and Technology, Harbin Institute of Technology, Harbin, Heilongjiang 150001, People's Republic of China; 이이이.org/0000-0001-99435677; Email: hezikai@hit.edu.cn

\section{Authors}

Jie Yu - School of Science, Harbin Institute of Technology, Shenzhen, Guangdong 518055, People's Republic of China

Huili Ma - Key Laboratory of Flexible Electronics \& Institute of Advanced Materials Jiangsu National Synergistic Innovation Center for Advanced Materials, Nanjing Tech University, Nanjing 211800, People's Republic of China; (1) orcid.org/0000-0003-0332-2999

Wenbin Huang - School of Science, Harbin Institute of Technology, Shenzhen, Guangdong 518055, People's Republic of China

Zhiwei Liang - School of Science, Harbin Institute of Technology, Shenzhen, Guangdong 518055, People's Republic of China

Kang Zhou - Hoffmann Institute of Advanced Materials, Shenzhen Polytechnic, Shenzhen, Guangdong 518055, People's Republic of China

Anqi Lv - Key Laboratory of Flexible Electronics \& Institute of Advanced Materials Jiangsu National Synergistic Innovation Center for Advanced Materials, Nanjing Tech University, Nanjing 211800, People's Republic of China

Complete contact information is available at: https://pubs.acs.org/10.1021/jacsau.1c00290

\section{Author Contributions}

OJ.Y. and H.M. contributed equally.

Notes

The authors declare no competing financial interest.

\section{ACKNOWLEDGMENTS}

This work was supported by the National Natural Science Foundation of China (21975061), the Natural Science Foundation of Guangdong Province (2019A1515011050), and the Shenzhen Fundamental Research Program (JCYJ20190806142403535, GXWD2020123015542700320200728150952003).

\section{REFERENCES}

(1) Zhao, W.; He, Z.; Tang, B. Z. Room-temperature phosphorescence from organic aggregates. Nat. Rev. Mater. 2020, 5 (12), 869885.

(2) Ma, H.; Peng, Q.; An, Z.; Huang, W.; Shuai, Z. Efficient and Long-Lived Room-Temperature Organic Phosphorescence: Theoretical Descriptors for Molecular Designs. J. Am. Chem. Soc. 2019, 141 (2), 1010-1015.

(3) Wang, J.; Gu, X.; Ma, H.; Peng, Q.; Huang, X.; Zheng, X.; Sung, S. H. P.; Shan, G.; Lam, J. W. Y.; Shuai, Z.; Tang, B. Z. A facile strategy for realizing room temperature phosphorescence and single molecule white light emission. Nat. Commun. 2018, 9 (1), 2963.

(4) Shi, H.; Song, L.; Ma, H.; Sun, C.; Huang, K.; Lv, A.; Ye, W.; Wang, H.; Cai, S.; Yao, W.; Zhang, Y.; Zheng, R.; An, Z.; Huang, W. Highly Efficient Ultralong Organic Phosphorescence through Intramolecular-Space Heavy-Atom Effect. J. Phys. Chem. Lett. 2019, 10 (3), 595-600.

(5) Xiong, Y.; Zhao, Z.; Zhao, W.; Ma, H.; Peng, Q.; He, Z.; Zhang, X.; Chen, Y.; He, X.; Lam, J. W. Y.; Tang, B. Z. Designing Efficient and Ultralong Pure Organic Room-Temperature Phosphorescent Materials by Structural Isomerism. Angew. Chem., Int. Ed. 2018, 57 (27), 7997-8001.

(6) Lower, S. K.; El-Sayed, M. A. The Triplet State and Molecular Electronic Processes in Organic Molecules. Chem. Rev. 1966, 66 (2), 199-241.

(7) Kalyanasundaram, K.; Grieser, F.; Thomas, J. K. Room temperature phosphorescence of aromatic hydrocarbons in aqueous micellar solutions. Chem. Phys. Lett. 1977, 51 (3), 501-505.

(8) Thomas, J. K. Effect of structure and charge on radiationinduced reactions in micellar systems. Acc. Chem. Res. 1977, 10 (4), 133-138.

(9) Schulman, E. M.; Parker, R. T. Room temperature phosphorescence of organic compounds. The effects of moisture, oxygen, and the nature of the support-phosphor interaction. J. Phys. Chem. 1977, $81(20), 1932-1939$.

(10) Zhang, G.; Chen, J.; Payne, S. J.; Kooi, S. E.; Demas, J. N.; Fraser, C. L. Multi-emissive difluoroboron dibenzoylmethane polylactide exhibiting intense fluorescence and oxygen-sensitive room-temperature phosphorescence. J. Am. Chem. Soc. 2007, 129 (29), 8942-3.

(11) Kwon, M. S.; Yu, Y.; Coburn, C.; Phillips, A. W.; Chung, K.; Shanker, A.; Jung, J.; Kim, G.; Pipe, K.; Forrest, S. R.; Youk, J. H.; Gierschner, J.; Kim, J. Suppressing molecular motions for enhanced room-temperature phosphorescence of metal-free organic materials. Nat. Commun. 2015, 6, 8947.

(12) Horie, K.; Mita, I. Photochemistry in polymer solids. Decay of benzophenone phosphorescence in poly(methyl methacrylate). Chem. Phys. Lett. 1982, 93 (1), 61-65.

(13) Valerii, L. E. Energy transfer in organic systems involving the triplet state III. rigid solution and crystals. Sov. Phys. Usp. 1963, 6 (3), 333.

(14) Yuan, W. Z.; Shen, X. Y.; Zhao, H.; Lam, J. W. Y.; Tang, L.; Lu, P.; Wang, C.; Liu, Y.; Wang, Z.; Zheng, Q.; Sun, J. Z.; Ma, Y.; Tang, B. Z. Crystallization-Induced Phosphorescence of Pure Organic Luminogens at Room Temperature. J. Phys. Chem. C 2010, 114 (13), 6090-6099.

(15) Bergamini, G.; Fermi, A.; Botta, C.; Giovanella, U.; Di Motta, S.; Negri, F.; Peresutti, R.; Gingras, M.; Ceroni, P. A persulfurated benzene molecule exhibits outstanding phosphorescence in rigid environments: from computational study to organic nanocrystals and OLED applications. J. Mater. Chem. C 2013, 1 (15), 2717-2724.

(16) Fermi, A.; Bergamini, G.; Peresutti, R.; Marchi, E.; Roy, M.; Ceroni, P.; Gingras, M. Molecular asterisks with a persulfurated benzene core are among the strongest organic phosphorescent emitters in the solid state. Dyes Pigm. 2014, 110, 113-122.

(17) Bilen, C. S.; Harrison, N.; Morantz, D. J. Unusual room temperature afterglow in some crystalline organic compounds. Nature 1978, 271 (5642), 235-237. 
(18) Bolton, O.; Lee, K.; Kim, H. J.; Lin, K. Y.; Kim, J. Activating efficient phosphorescence from purely organic materials by crystal design. Nat. Chem. 2011, 3 (3), 205-210.

(19) Fermi, A.; Bergamini, G.; Roy, M.; Gingras, M.; Ceroni, P. Turn-on phosphorescence by metal coordination to a multivalent terpyridine ligand: a new paradigm for luminescent sensors. J. Am. Chem. Soc. 2014, 136 (17), 6395-400.

(20) Gu, L.; Shi, H.; Bian, L.; Gu, M.; Ling, K.; Wang, X.; Ma, H.; Cai, S.; Ning, W.; Fu, L.; Wang, H.; Wang, S.; Gao, Y.; Yao, W.; Huo, F.; Tao, Y.; An, Z.; Liu, X.; Huang, W. Colour-tunable ultra-long organic phosphorescence of a single-component molecular crystal. Nat. Photonics 2019, 13 (6), 406-411.

(21) Wang, X.; Shi, H.; Ma, H.; Ye, W.; Song, L.; Zan, J.; Yao, X.; Ou, X.; Yang, G.; Zhao, Z.; Singh, M.; Lin, C.; Wang, H.; Jia, W.; Wang, Q.; Zhi, J.; Dong, C.; Jiang, X.; Tang, Y.; Xie, X.; Yang, Y.; Wang, J.; Chen, Q.; Wang, Y.; Yang, H.; Zhang, G.; An, Z.; Liu, X.; Huang, W. Organic phosphors with bright triplet excitons for efficient X-ray-excited luminescence. Nat. Photonics 2021, 15 (3), 187-192.

(22) Wu, H.; Zhou, Y.; Yin, L.; Hang, C.; Li, X.; Agren, H.; Yi, T.; Zhang, Q.; Zhu, L. Helical Self-Assembly-Induced Singlet-Triplet Emissive Switching in a Mechanically Sensitive System. J. Am. Chem. Soc. 2017, 139 (2), 785-791.

(23) Lee, D.; Bolton, O.; Kim, B. C.; Youk, J. H.; Takayama, S.; Kim, $\mathrm{J}$. Room temperature phosphorescence of metal-free organic materials in amorphous polymer matrices. J. Am. Chem. Soc. 2013, 135 (16), 6325-9.

(24) Gu, L.; Shi, H.; Gu, M.; Ling, K.; Ma, H.; Cai, S.; Song, L.; Ma, C.; Li, H.; Xing, G.; Hang, X.; Li, J.; Gao, Y.; Yao, W.; Shuai, Z.; An, Z.; Liu, X.; Huang, W. Dynamic Ultralong Organic Phosphorescence by Photoactivation. Angew. Chem., Int. Ed. 2018, 57 (28), 8425-8431.

(25) Wang, Y.; Yang, J.; Fang, M.; Yu, Y.; Zou, B.; Wang, L.; Tian, Y.; Cheng, J.; Tang, B. Z.; Li, Z. Förster Resonance Energy Transfer: An Efficient Way to Develop Stimulus-Responsive Room-Temperature Phosphorescence Materials and Their Applications. Matter 2020, 3 (2), 449-463.

(26) Kabe, R.; Notsuka, N.; Yoshida, K.; Adachi, C. Afterglow Organic Light-Emitting Diode. Adv. Mater. 2016, 28 (4), 655-660.

(27) Wang, Y.; Gao, H.; Yang, J.; Fang, M.; Ding, D.; Tang, B. Z.; Li, Z. High Performance of Simple Organic Phosphorescence Host-Guest Materials and their Application in Time-Resolved Bioimaging. Adv. Mater. 2021, 33 (18), 2007811.

(28) Dang, Q.; Jiang, Y.; Wang, J.; Wang, J.; Zhang, Q.; Zhang, M.; Luo, S.; Xie, Y.; Pu, K.; Li, Q.; Li, Z. Room-Temperature Phosphorescence Resonance Energy Transfer for Construction of Near-Infrared Afterglow Imaging Agents. Adv. Mater. 2020, 32 (52), 2006752.

(29) Zhen, X.; Tao, Y.; An, Z.; Chen, P.; Xu, C.; Chen, R.; Huang, W.; Pu, K. Ultralong Phosphorescence of Water-Soluble Organic Nanoparticles for In Vivo Afterglow Imaging. Adv. Mater. 2017, 29 (33), 1606665.

(30) Garain, S.; Kuila, S.; Garain, B. C.; Kataria, M.; Borah, A.; Pati, S. K.; George, S. J. Arylene Diimide Phosphors: Aggregation Modulated Twin Room Temperature Phosphorescence from Pyromellitic Diimides. Angew. Chem., Int. Ed. 2021, 60 (22), 12323-12327.

(31) Yin, Z.; Gu, M.; Ma, H.; Jiang, X.; Zhi, J.; Wang, Y.; Yang, H.; Zhu, W.; An, Z. Molecular Engineering through Control of Structural Deformation for Highly Efficient Ultralong Organic Phosphorescence. Angew. Chem., Int. Ed. 2021, 60 (4), 2058-2063.

(32) Tang, X.; Cui, L. S.; Li, H. C.; Gillett, A. J.; Auras, F.; Qu, Y. K.; Zhong, C.; Jones, S. T. E.; Jiang, Z. Q.; Friend, R. H.; Liao, L. S. Highly efficient luminescence from space-confined charge-transfer emitters. Nat. Mater. 2020, 19 (12), 1332-1338.

(33) Wada, Y.; Nakagawa, H.; Matsumoto, S.; Wakisaka, Y.; Kaji, H. Organic light emitters exhibiting very fast reverse intersystem crossing. Nat. Photonics 2020, 14 (10), 643-649.

(34) Woon, K. L.; Yi, C. L.; Pan, K. C.; Etherington, M. K.; Wu, C. C.; Wong, K. T.; Monkman, A. P. Intramolecular Dimerization
Quenching of Delayed Emission in Asymmetric D-D'-A TADF Emitters. J. Phys. Chem. C 2019, 123 (19), 12400-12410.

(35) Chen, X. K.; Bakr, B. W.; Auffray, M.; Tsuchiya, Y.; Sherrill, C. D.; Adachi, C.; Bredas, J. L. Intramolecular Noncovalent Interactions Facilitate Thermally Activated Delayed Fluorescence (TADF). J. Phys. Chem. Lett. 2019, 10 (12), 3260-3268.

(36) Tsujimoto, H.; Ha, D.-G.; Markopoulos, G.; Chae, H. S.; Baldo, M. A.; Swager, T. M. Thermally Activated Delayed Fluorescence and Aggregation Induced Emission with Through-Space Charge Transfer. J. Am. Chem. Soc. 2017, 139 (13), 4894-4900.

(37) Lin, J.-A.; Li, S.-W.; Liu, Z.-Y.; Chen, D.-G.; Huang, C.-Y.; Wei, Y.-C.; Chen, Y.-Y.; Tsai, Z.-H.; Lo, C.-Y.; Hung, W.-Y.; Wong, K.-T.; Chou, P.-T. Bending-Type Electron Donor-Donor-Acceptor Triad: Dual Excited-State Charge-Transfer Coupled Structural Relaxation. Chem. Mater. 2019, 31 (15), 5981-5992.

(38) Hu, J.; Li, Q.; Wang, X.; Shao, S.; Wang, L.; Jing, X.; Wang, F. Developing Through-Space Charge Transfer Polymers as a General Approach to Realize Full-Color and White Emission with Thermally Activated Delayed Fluorescence. Angew. Chem., Int. Ed. 2019, 58 (25), 8405-8409.

(39) Shao, S.; Wang, L. Through-space charge transfer polymers for solution-processed organic light-emitting diodes. Aggregate 2020, 1 (1), 45-56.

(40) Yang, Z.; Mao, Z.; Zhang, X.; Ou, D.; Mu, Y.; Zhang, Y.; Zhao, C.; Liu, S.; Chi, Z.; Xu, J.; Wu, Y. C.; Lu, P. Y.; Lien, A.; Bryce, M. R. Intermolecular Electronic Coupling of Organic Units for Efficient Persistent Room-Temperature Phosphorescence. Angew. Chem., Int. Ed. 2016, 55 (6), 2181-5.

(41) Ward, J. S.; Nobuyasu, R. S.; Batsanov, A. S.; Data, P.; Monkman, A. P.; Dias, F. B.; Bryce, M. R. The interplay of thermally activated delayed fluorescence (TADF) and room temperature organic phosphorescence in sterically-constrained donor-acceptor charge-transfer molecules. Chem. Commun. 2016, 52 (12), 26122615.

(42) He, Z.; Zhao, W.; Lam, J. W. Y.; Peng, Q.; Ma, H.; Liang, G.; Shuai, Z.; Tang, B. Z. White light emission from a single organic molecule with dual phosphorescence at room temperature. Nat. Commun. 2017, 8 (1), 416.

(43) Zhao, W.; Cheung, T. S.; Jiang, N.; Huang, W.; Lam, J. W. Y.; Zhang, X.; He, Z.; Tang, B. Z. Boosting the efficiency of organic persistent room-temperature phosphorescence by intramolecular triplet-triplet energy transfer. Nat. Commun. 2019, 10 (1), 1595.

(44) Chen, C.; Chi, Z.; Chong, K. C.; Batsanov, A. S.; Yang, Z.; Mao, Z.; Yang, Z.; Liu, B. Carbazole isomers induce ultralong organic phosphorescence. Nat. Mater. 2021, 20 (2), 175-180.

(45) Wu, Z.; Nitsch, J.; Schuster, J.; Friedrich, A.; Edkins, K.; Loebnitz, M.; Dinkelbach, F.; Stepanenko, V.; Würthner, F.; Marian, C. M.; Ji, L.; Marder, T. B. Persistent Room Temperature Phosphorescence from Triarylboranes: A Combined Experimental and Theoretical Study. Angew. Chem., Int. Ed. 2020, 59 (39), 1713717144.

(46) Wu, Z.; Nitsch, J.; Marder, T. B. Persistent Room-Temperature Phosphorescence from Purely Organic Molecules and MultiComponent Systems. Adv. Opt. Mater. 2021, 2100411.

(47) Zhang, Z.-Y.; Liu, Y. Ultralong room-temperature phosphorescence of a solid-state supramolecule between phenylmethylpyridinium and cucurbit 6 uril. Chem. Sci. 2019, 10 (33), 7773-7778.

(48) Kim, J. U.; Park, I. S.; Chan, C. Y.; Tanaka, M.; Tsuchiya, Y.; Nakanotani, H.; Adachi, C. Nanosecond-time-scale delayed fluorescence molecule for deep-blue OLEDs with small efficiency rolloff. Nat. Commun. 2020, 11 (1), 1765.

(49) Etherington, M. K.; Gibson, J.; Higginbotham, H. F.; Penfold, T. J.; Monkman, A. P. Revealing the spin-vibronic coupling mechanism of thermally activated delayed fluorescence. Nat. Commun. 2016, 7, 13680.

(50) Turro, N. J.; Ramamurthy, V.; Scaiano, J. C. Modern Molecular Photochemistry of Organic Molecules; Viva Books, University Science Books: 2017. 